METABOLIC ENGINEERING

\section{Polyphenols on order}

Met. Eng., published online 14 June 2013;

doi:10.1016/j.ymben.2013.06.001

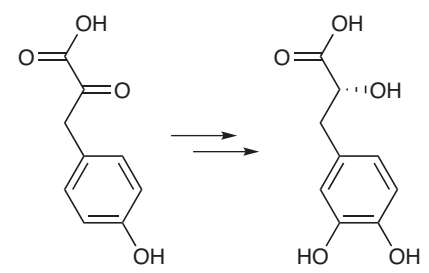

Salvianic acid $\mathrm{A}$ is an efficient antioxidant and possibly has other biological roles, but limited availability and a lack of known biosynthetic enzymes of this polyphenolic acid has stymied a full understanding of its functions. Yao et al. noted that related aromatic compounds have been produced enzymatically in cells engineered for tyrosine production, but these studies focused primarily on tyrosine-derived compounds. The authors anticipated that rerouting of the tyrosine precursor, 4-hydroxyphenylpyruvate (4-HPP), in Escherichia coli would provide a more direct route to the desired product. The authors tested D-lactate dehydrogenase and two known activated mutants as catalysts for the chiral reduction of the ketone group and identified the flavoenzyme 4-hydroxyphenylacetate 3-hydroxylase hpaBC as likely to insert a 3-hydroxy group on the phenyl ring; expression of the pair of enzymes returned $259.27 \mathrm{mg} \mathrm{l}^{-1}$ salvianic acid A from a strain fed with $500 \mathrm{mg} \mathrm{l}^{-1} 4$-HPP. The authors separately adopted existing strategies to engineer tyrosine biosynthesis to optimize yield of the 4-HPP precursor, with the best success observed in a strain that overexpressed three enzymes that synthesize precursors very early in the pathway. Combination of the two strategies in fed-batch cultures led to an average titer of $7.1 \mathrm{~g} \mathrm{l}^{-1}$, or $0.47 \mathrm{~mol} / \mathrm{mol}$ glucose, with salvianic acid A toxicity limiting further accumulation. This artificial route provides a new source for a plant polyphenol. CG

\section{MOLECULAR RECOGNITION}

\section{Mimicry origins}

\section{J. Immunol. 191, 456-463 (2013)}

Several autoimmune diseases manifest as a result of molecular mimicry, where completely unrelated molecules, such as an allergen and a self-peptide, share a common receptor. Methyl $\alpha$-D-mannopyranoside and a dodecapeptide, DVFYPYPYASGS, can be interchangeably bound by the lectin concanavalin A and a monoclonal antibody $2 \mathrm{D} 10$ as well as by polyclonal sera. For concanavalin A, binding of the two ligands occurs at different sites, so the differences in molecular recognition of the two ligands are not attributable to their different structures per se. For 2D10, it has been suggested from thermodynamic and in silico studies that 2D10 uses a flexible binding site that can accommodate both antigens. To test this proposal, Tapryal et al. solved the crystal structures of a single-chain variable fragment of 2D10 in an antigen-free state as well as in complexes with the carbohydrate and dodecapeptide. Surprisingly, they found no

\title{
DRUG RESISTANCE
}

\section{Starving for ATP}

Therapeutics that target activated mutants of the kinase RAF, such as vemurafenib, are initially effective in malignant melanoma, but tumors rapidly acquire resistance to the drug. A slow-replicating subpopulation of melanoma cells, marked by the expression of the histone 3 lysine 4 demethylase JARID1B, has been previously discovered. Roesch et al. now report that this subpopulation drives vemurafinib resistance by increasing cellular reliance on mitochondrial oxidative phosphorylation for energy production. In culture, exposure to vemurafenib increased the proportion of these slow-cycling cells in the dish. Similar results were achieved with different drugs, including cisplatin and bortezomib, indicating that this JARID1B-expressing subpopulation may contribute to resistance to multiple drugs. Quantitative proteomic profiling comparing JARID1B-expressing with nonexpressing cells revealed enriched expression of mitochondrial proteins involved in energy metabolism in the presence of JARID1B. Small molecule-mediated inhibition of respiratory chain enzymes decreased ATP production and increased cell death of JARID1B-expressing cells in culture and in three-dimensional colony-forming assays. Combinatorial treatment with vemurafinib and inhibitors of oxidative phosphorylation prevented the expansion of the JARID1B subpopulation and enhanced tumor cell death in culture and in xenograft tumor models. Taken together, these data suggest that oxidative phosphorylation drives the expansion of a drug-resistant subpopulation of tumor cells in melanoma and suggests that compounds that target this energy production system could help to eliminate this cell population.

overall structural differences in the antibody fragments, suggesting a rigid binding site that does not change upon antigen binding. The authors also found that the carbohydrate bound the antibody primarily through polar interactions, whereas the peptide bound via different polar interactions but also by hydrophobic interactions. Therefore, molecular mimicry arises by neither the chemical nature nor the equivalence of the interactions nor with any role for conformational changes at the antigenbinding site but is achieved via plasticity of the antigen-antibody interactions.

\section{X-INACTIVATION}

\section{RNA eviction notice \\ Cell 153, 1537-1551 (2013)}

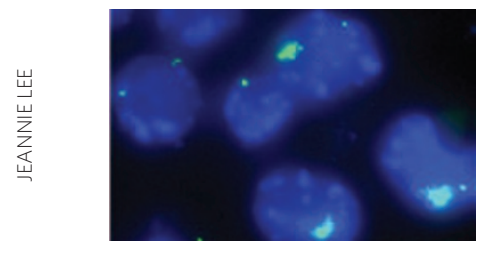

X-chromosome inactivation (XCI) leads to the transcriptional silencing of one $\mathrm{X}$ chromosome, thereby balancing the dosage of X-linked genes between XX females and $\mathrm{XY}$ males. The process is coordinated by the $\mathrm{X}$-inactivation center, which includes four genes that encode long noncoding RNAs: Xist, Tsix, Xite and Jpx. The chromosome selected for inactivation transcribes Xist RNA, which localizes on that chromosome as an 'Xist cloud' and marks it for silencing. Previous studies had shown that Xist expression is positively regulated by $J p x$, but how this was linked to initiation of XCI remained unclear. Sun et al. show that XCI is initiated when Jpx RNA titrates away a repressor protein from the Xist promoter, thereby activating Xist RNA expression. CTCF, an autosomal protein with known binding sites in the Xist promoter, was shown to repress Xist transcription, and CTCF-mediated repression could be relieved by enhanced Jpx RNA expression. Jpx RNA specifically binds CTCF in vitro, and UV crosslinked RNA immunoprecipitation analysis confirmed the binding interaction within cells. Gene reporter assays in cells further established that overexpression of CTCF repressed Xist expression mediated by the $\mathrm{P} 2$ promoter element, whereas overexpression of Jpx enhanced Xist transcription. The resulting mechanism, in which the autosomal CTCF repressor is regulated by the X-linked Jp $x$ RNA, provides a molecular framework for sensing the 'X-to-autosome' ratios in cells, a concept that underpins current genetic models of XCI. TLS 